NASÁ Technical Memorandum 89436

\title{
An Astrometric Facility for Planetary Detection on the Space Station
}

Kenji Nishioka, Jeffrey D. Scargle, and John J. Givens (NASA-TH-89436) AN ASTECUETEIC FACILITY FOR
ELASETARY DETECTICA CA THE SELCE STATICA
(SASA) $16 \mathrm{p}$

April 1987
$N 87-2 C 841$

Unclas

G3/89 45400 
NASA Technical Memorandum 89436

\section{An Astrometric Facility for Planetary Detection on the Space Station}

Kenji Nishioka, Jeffrey D. Scargle, John J. Givens, Ames Research Center, Moffett Field, California

April 1987

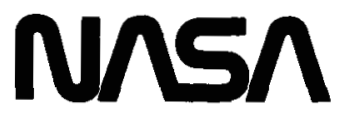

National Aeronautics and

Space Administration 
An astrometric facility for planetary detection on the Space Station

Kenji Nishioka, Jeffrey D. Scargle, and John J. Givens

ATF Sundy Office, NASA Ames Research Center, Moffett Field, CA 94035

\section{Abstract}

An Astrometric Telescope Facility (ATF) for planetary detection is being studied as a potential Space Station initial operating capability payload. The primary science objective of this mission is the detection and study of planetary systems around other stars. In addition, the facility will be capable of other astrometric measurements such as stellar motions of other galaxies and highly precise direct measurement of stellar distances within the Milky Way Galaxy. This paper summarizes the results of a recently completed $\wedge$ if preliminary systems definition study. Results of this study indicate that the preliminary concept for the facility is fully capable of meeting the science objectives without the development of any new technologies.

This preliminary systems study started with the following basic assumptions: 1 ) the facility will be placed in orbit by a single Shuttle launch, 2) the Space Station will provide a coarse pointing system, electrical power, communications, assembly and checkout, maintenance and refurbishment services, and 3 ) the facility will be operated from a ground facility. With these assumptions and the science performance requirements a preliminary "strawman" facility was designed. The strawman facility design with a prime-focus telescope of 1.25 -1m aperture, $f$-ratio of 1.3 and a single prime-f(cus instrument was chosen to ninimize random and systemmatic errors. Tolal facility mass is $5100 \mathrm{~kg}$ and overall dimensions are $1.85-\mathrm{m}$ diam by $21.5-\mathrm{m}$ long. $A$ simple straightforward operations approach has been developed lor $\Lambda \mathrm{TF}$. $\AA$ real-time facility control is not nonmilly required, but does maintain a near real-time ground monitoring capatbility for facility and science data stream on a full-time basis. Facility observational sequences are nomally loaded once a week. In addition, he preliminary system is designed to be fail-safe and single-fault folerant. Routine inferactions by the Space Station crew with ATF will mot be mecessary, but onboard controls are provided for crew override as required for emergencies and maintenance.

\section{Introduction}

The $\Lambda$ strometric 'Telescope Facility (ATF) is a spaceborne observatory proposed for the Space Station as an initial operating capability (IOC) payload. The primary objective of the ATF is the search for extrasolar planetary systems and detailed investigations of any discovered systems. In addition, it will have the capability of conducting other astrophysics investigations, e.g., measuring precise distances and motions of stars within our Milky Way Galaxy.

The current ATF concept is the result of many scientific workshops ${ }^{1-5}$ and several systems studies, ${ }^{6-8}$ starting in 1971 , that examined, extrasolar planetary detection methods and their feasibility. These workshops were concerned primarily with assessing the scientific worth of extrasolar planetary detection and identifying the objectives, goals, and methods that could be used for the search. These activities were conducted by space scientists and engineers who established the scientific and engineering feasiblity for such searches, and identified the astrometric method as the most promising for current initial investigations. Mutual interests in the science of planet and star formation and in a program of extrasolar planetary detection and study by the University of Arizona and NASA Ames Research Center. led to an agreement between these institutions to work jointly on the development of the ATF project described in this project. As a start, a joint team made up of members from the University of Arizona (includes the University of Pittsburgh--The Allegheny Observatory, and the Unversity of California at San Diego) and NASA Ames Research Center conducted this ATF Preliminary System Definition Study in 1986. The purposes of the studly were to:

1. Define mission and system requirements.

2. Define a strawman system concept for the facility at the Pre-Phase A level.

3. Define the need for additional trade studies or technology development.

For this study, the ATF as a Space Station payload has been assumed to have dedicated use of a Space Station provided coarse pointing system (CPS), use of Space Station utilities (power and communications), meet Space Station constraints, and use of Space Station qualified hardware and software to the maximum extent possible. In addition, other existing space qualified hardware designs will be fully exploited in the ATF design

\section{Mission description and requirements}

\section{Science}

The primary objective of the ATF mission is the search for extrasolar planetary systems and the study of these planetary systcms. This objective, if successful, will either verify, modify, or refute current scientific theories about accretion of interplanetary clouds into stars and planets that are based on a singular observation, our solar system. Also the quintessential question, "Are we unique; alone?", will be answered.

The orderly nature of our solar system suggests that it originated from a single precursor object and not from a series of accidents. Present evidence suggests that it was formed from a rotating cloud which, when acted on by viscous effects and self gravitation. resulted in the overall configuration of the Solar System. This theory is consistent with the observation that planetary composition varies with the distance from the Sun and that planetary masses are correlated with the cosmic abundance of material from which they are made. If this theory is correct, then planetary systems are fomed as a natural consequence of star fommation, at least for single stars, and it is believed that our Solar System is not unique. On the other hand, if a statistically significant survey of stars fails to discover planetary systems, this will show our system to be unique, and existing theories would have to be re-examined. 
The basic ATF goals were defined by the scientific workshops, ${ }^{1-5}$ which are to provide a facility with the necessary sensitivity to detect and study extrasolar planets with masses as small as 10 to 20 Earth masses and that negative results would be scientifically significant, observed on the order of 100 stars (out to 10 parsecs).

\section{Measurementapproach}

The ATF approach is to make astrometric measurements with respect to a frame of distant stars, to an accuracy which would show the reflex motion of a star as it and the associated planets orbit around the system's barycenter. To accomplish this, the ATF must have the capability of detecting relative motion of $10^{-5}$ arcsec to allow useful observation and study of stars to a distance of 10 parsecs from the sun. In addition, the duration of the observations must continue for 10 to $20 \mathrm{yr}$, which are commensurate with expected orbital periods of planets.

The ATF measurement approach (Figure 1) extracts metric information from the star images by passing a Ronchi ruling (a series of tramsparent and opaque lines) across the telescope focal plane, thus modulating the star light (signals) to sensors located beyond the ruling. The distance between the target and reference star images is determined accurately (10 microarcsec) by summing the number of ruling lines between images and by adding the fractional ruling line extracted by determining the relative phase differences of the modulated signals. Relative stellar motions can therefore be determined from the accumulated motions of the stars determined by plotting the phase shifts between images measured over periods commensurate with planetary orbital periods, 10 to $20 \mathrm{yr}$.

It is important to remenber that in the context of planetary detection, the measurement being made is the star's change in position, not absolute (inertial) position itself. Thus the ATFs 10-microarcsec accuracy refers to the accuracy in the knowledge of the star's change in position through a series of observations, rather than in the star's absolute position. Many general astrometric investigations, apart from planetary detection, also require measurement of an object's change in position over time and are well suited to the ATF.

This study and the work done prior to its initiation show that the ATF strawman system can achieve the required measurement accuracy. Analyses included consideration of both systematic and random errors. Effect of random errors on the final result can be significantly reduced by increasing the observation time. Systematic errors on the other hand must be identified and removed either by design, or system calibration and in the data reduction process.

Table 1 summarizes the identified systematic errors with how they could be resolved. As seen from the list, some of these errors can be controlled by basic design, while others will be controlled by system calibration or accounted for in the data reduction.

Expected major sources of random errors for the ATF strawman design have been identified and analysed. Based on the results to date, it appears that the ATF mission requirements can be met. The following is a discussion of the analyses showing the impact of these errors on integration time.

The prime requirement for measurement accuracy is to find the phase of the modulated signal (light intensity vesus time) from the start with a relative accuracy of 10 microarcsec. For a normal distribution of photons from the star, the maximum achievable measurement accuracy is

$$
\mathrm{s}=\mathrm{s}_{\mathrm{O}}+\mathrm{N}^{0.5}
$$

where $s_{\mathrm{O}}$ is the size of the diffraction image and $\mathrm{N}$ is the total number of photons detected. Since the diffraction limited image for the telescope is on the order of 0.1 arcsec, the equation shows that $10^{8}$ photons are required for the measurement exclusive of any error sources. The effects of random errors add in the following way

$$
\left.s^{2}=s_{o}^{2}+s_{o}^{2} \sum \underset{\substack{i=1}}{\left(s_{i} / s_{o}\right.}\right)^{2}
$$

where the $s_{i}$ are the standard deviations for the various random error sources. The recognized error sources (Table 2 ) combined with the efficiency factor associated with various losses in the system (Table 3) increase the integration time required for one determination of a target star position relative to the ideal case by a factor of about 1160 . Note that the total number of photons required can be accumulated over multiple integration periods. Table 4 shows the total time required for one observation (integration time for required astrometric accuracy); it is shown as a function of galactic latitude because of the systematic dependence of reference star brightness with galactic latitude. As indicated in Table 4 the observation times for target stars up to about $30^{\circ}$ galactic latitude are commensurate with available mission observing time.

\section{Mission}

The ATF is planned as a Space Station IOC payload, launched by the Space Transportation System (STS). The strawman facility was sized to best accomplish the mission science goals within the constraint that it be capable of being placed in orbit by a single STS launch. The resulting design essentially uses all of the available volumetric payload bay volume of the STS and thus requires a dedicated launch unless the three pieces that make up the ATF are launched separately. If the ATF pieces are launched separately, each piece will be stored on the Station until all the pieces have been launched and the ATF can be assembled. The baseline plan is to assemble the ATF in the Space Station service bay, then move it to the mounting location where it would be attached to the CPS. Subsequent to the initial assembly, the facility will be operated from the ground, requiring no further on-orbit manned activity except for maintenance and repair of failed hardware or for possible preemptive shutdown by the Space Station crew. In the event the service bay is not part of the IOC configuration, the ATF could be assembled directly onto the CPS at the mounting location. 
The ATF has been designed for Space Station accommodation on an extension attached to the aft face on the north end of the upper science boom. Figure 2 shows the baseline location and mounting configuration used for the study. Although this is the most desirable operational location, the study indicated that the ATF mission could be accomplished while mounted at other locations with some changes to the CPS configuration. Location on the station and the ATF hardware design have been selected to provide repeated full-sky coverage over the life of the mission. The full-sky viewing is achieved while still meeting the avoidance constraints: greater than $30^{\circ}$ from the $S u n$ and Earth, greater than $10^{\circ}$ from the Moon, and greater than $90^{\circ}$ from the velocity vector. With this configuration, it will be possible to investigate 100 target stars in the visual magnitude range of -1.5 to +13.5 against fields with reference stars down to +15 visual magnitude.

The ATF mission can tolerate the present Space Station specified levels of contamination. However, the team is aware of studies indicating that these contamination levels are optimistic and that they could be considerably higher. If this proves correct, depending on the revised levels, the contamination problem for ATF will be reassessed.

Coherent mechanical jitter at the ruling frequency or at one-half or twice its frequency (at to decreasing degrees at higher or lower harmonics) can be detrimental to measurement accurracy and is of concern to ATF. These coherent vibrations are not expected but if they occur, they will distort the signal trace of the modulated star light signal and cause a shift in the deduced centroid, and thus affect the measurement accuracy of the phase differences between stars. If the vibrational frequency is known, this problem can be avoided by changing the ruling frequency. Because the mechanical vibration environment on the Space Station at the upper science boom is undefined, the ATF strawman design as a precaution, includes a vibration-isolation mechanism. Analyses show that the strawman ATF vibrationisolation design meets the mission requirements for the maximum currently anticipated station vibration.

The ATF design mission lifetime is $20 \mathrm{yr}$ with a minimum time between maintenance of $5 \mathrm{yr}$. No expendables are used by the $\Lambda$ TF. The minimum maintenance period was established consistent with our current experience with space-qualified flight hardware which is typically $5 \mathrm{yr}$. Experience has shown that most hardware normally exceed their qualification life, therefore, the anticipated 5 -yr maintenance should be required less frequently than specified. One of the major attributes of the Space Station is the practicability of planned maintenance. This eliminates the need to provide 20-yr hardware lifetimes.

\section{System description}

The ATF system is composed of six subsystems (optics, structure, thermal control, command and data, pointing and control, and power and harness) plus the focal plane instrument (FPI) (Ronchi Ruling and drive assembly, folding and field optics, multichannel astrometric photometer and visible imager). These elements are integrated into a system designed to meet the mission and basic science requirements to measure relative star motions with an accuracy of 10 microarcsec. One rack of ATF electronic equipment designated as the ATF control console will be located inside a Space Station pressurized module, and it will contain all required control functions for on-board safing plus a computer for contingency ATF operation and data reduction. The on-board data-reduction capability would be used only if there were significant periods (weeks) when the station could not support the full ATF downlink data rate. The baseline is to transmit all of the raw data to the ground for archiving and reduction.

Figure 3 shows the overall layout of the ATF. The telescope is designed as a prime-focus telescope with a 1.25-m-diam parabolic primary mirror and a focal ratio of 13. The Ronchi ruling is located at the prime focus. A bar across the front of the telescope tube supports the ruling and the post focal optics which direct the beam to the side of the tube where it is refocused and magnified at the secondary focus located at the multichannel astrometric photometer (MAP). Also shown in the figure is the sunshade and protective cover. The telescope is wrapped with a thermal blanket to minimize temperature changes associated with varying solar and Earth illumination conditions. Overall diameter of the telescope assembly including the blanket is approximately $1.85 \mathrm{~m}$. The telescope is held at its center of gravity in the ATF vibration isolation/vernier pointing system (VIVPS) which is, in turn, attached to the Space Station CPS. The VIVPS isolates the telescope from the Space Station vibrations and provides a second level of pointing (fine pointing) to reach the mission pointing accuracy requirement of 1 arcsec (CPS capability is limited to approximately $30 \mathrm{arcsec}$ ). Also included in this assembly is a mechanism to rotate the telescope

$\pm 180^{\circ}$ about the optical axis. Electronics units are located on a thermally isolated plate at the back end of the telescope tube and at the front of the tube in the region of the FPI. Simple passive radiators are used to reject heat from the electronics and to keep the detectors cool. The mass of the ATF mounted to the CPS is $5100 \mathrm{~kg}$.

Figure 4 is a schematic of the ATF system showing the systems approach to operational reliability through redundancy and the electrical/electronic interface with the station. Redundancy has been implemented by providing two independent strings of electronics, while cross-strapping is used only for three components, the FPI, the visible imager, and the gyroscope. Engineering review of the FPI, indicated that it was impractical to make it redundant and as seen later, the instrument does contain important elements of redundancy. The gyros are already internally redundant. The ATF Station interface has been kept to a standard data and power interface. The maximum data rate is $1.75 \mathrm{Mbps}$. Power levels are $1400 \mathrm{~W}$ average, and $2500 \mathrm{~W}$ maximum.

The system is designed to be fail-safe and single-fault tolerant. It is assumed that the CPS operational control software will include safety features to prevent telescope pointing which could physically damage the telescope, Space Station, or other adjacent science payload. Based on this assumption, the potential critical conditions for ATF are pointing the telescope into the Sun, exposure to high levels of contamination, or significant over- or under-voltage conditions. An aperture cover will provide a second level of protection against improper pointing or contamination and is designed to close automatically, without or with extemal power, as when power to ATF is lost or when critical mispointing is detected by onboard sun sensors. The Space Station is expected to provide a contamination waming signal for planned or inadvertent contamination events. The ATF system is protected from anomalous voltage conditions by the power subsystem electronics. Noncritical failures have been accommodated by the redundancy approach described above. This approach works as follows, should a failure occur in one string, the system would be switched to the other and continue to operate. Replacement of the failed unit would he scheduled for the next convenient opportunity from an overall Space Station standpoint, with the ATF continuing to operate normally. 
The primary mirror is a parabolic design with a focal length of $16.25 \mathrm{~m}$ and diameter of $1.25 \mathrm{~m}$ is used to focus the field at the ruling. For best performance, the strawman design maximized these dimensions while meeting the the STS payload bay envelope constraint. The clear aperture field of view of the telescope is $10 \mathrm{arcmin}$. This design is pupilar and therefore resistant fo metric errors caused by spatial variations in the mirror performance. This results from the entire mirmo being illuminated equally by all the stars in the field of view and thus all reflected images are equally affected. However, such a system could have significant comatic and chsomatic problems. The ATF design has minimized the coma by using a long focal ratio (F 13) and the chromatic by optimizing the ruling constant and by dividing the spectral region into two ranges $(0.4$ to 0.6 and 0.6 to $0.8 \mathrm{~m})$ each with its own dedicated photodetector.

The image of the star field focused at the Ronchi ruling by the primary mirror is modulated by the ruling and this modulated light signal is directed to the side of the tube by a diagonal mirror and is magnified two and a half times $(5$ to $12.5 \mathrm{~cm})$ by a relay lens assembly. This provides a larger working image area for the 32 movable pickup assemblies of the MAP.

The MAP component for the FPI has been demonstrated by a similar multi-head instrument, nicknamed "Medusa," currently in use for spectroscopic investigations at Steward Observatory, University of Arizona. Figure 5 shows the design of the ATF instrument based on this experience. A notable difference is that for the ATF instrument the pickup drives are mounted at two levels to permit easier packaging of the 32 pickups while that for the University of Arizona instrument is at a single level. Also shown is the visible imager location at the back of the MAP. The imager will use a standard $512 \times 512$ CCD (charge-coupled device) with three lens groups (telephoto, normal, and wide angle). These lenses will provide the capability of imaging the telescope objective for diagnostic purposes by providing a telescope field-ofview image, or a magnified image of a single star.

\section{Subsystems description}

\section{Structure and mechanisms}

The basic structure of the teiescope is a tube of graphite-epoxy material formulated to minimize the axial coefficient of themal expansion. Where required, the tube will be reinforced locally with honeycomb material. The monocoque design is relatively straightforward to fabricate and will have few resonant modes. This is particularly important for ATF because the accuracy of the measurement is sensitive to image jitter as discussed earlier. Limiting the number of resonances thus reduces the frequency bands of concern. A NASTRAN model of the structure was developed during the study and the results of this model were used to determine the vibration- isolation requirements.

The Ronchi ruling assembly is shown in Figure 6. This assembly has been designed as a cartridge for ease of installation or replacement. The ruling rides in a set of bearings and is driven by a variable speed motor using a metal belt. Redundant motors have been incorporated in the design. The variable speed drive provides the capability to modulate the frequency for the range 10 to $100 \mathrm{~Hz}$.

The primary mirror mount holds the mirror kinematically and includes a mechanism which will allow the system to be collimated and focused on orbit with motions in the axial, tilt and radial directions. It also provides a mechanism for locking the mirtor in place during the launch phase. The locking mechanism is designed to be released manually during the initial on-orbit assembly process.

The aperture cover is designed to close automatically to protect the optical system and FPI in case of anomalous conditions including power failure. The cover is designed to fold in the middle so that the inner surface is protected from contamination when in the open position.

\section{Thermal control}

Passive thermal control will be used for the ATF with the exception of the primary mirror which will be heated to maintain a 20 oF temperature above ambient to minimize possibility of surface deposits and the electronicsto prevent freezing. All electronics including electric drives will be cooled by fixed passive radiators to reject heat, and heated if required. Temperature control of the telescope tube will depend on standard super insulation thermal blankets to minimize the tube wall temperature differences at any give time to about 8 oF. First order steady-state analyses, using a conservative value for the coefficient of thermal expansion for the graphite epoxy, show the telescope tube system has adequate dimensional stability to meet the optical alignment requirements for the calculated temperature extremes.

\section{Command and data}

A block diagram of the command and data subsystem and its interface with the Space Station data system is shown in Figure 7 . The electronic units used in this subsystem, with the exception of the signal conditioning unit and the data reduction processor, are Space Station units with minimal modification. This approach will minimize ATF project costs and ensure interface compatibility with the Space Station data system. ATF requirements are well within the capability of the Space Station unit design.

\section{Pointing and control}

A schematic of the pointing and control subsystem is shown in Figure 8. The precision gyro design selected for ATF is a flightproven unit while the star tracker, although based on units that have been built and tested for flight, have not flown at this time (It was scheduled for the ASTROS II mission for the summer of 1986.). The VIVPS will be designed and built for ATF. However, other systems applications with more severe requirements have now been built and tested successfully at the breadboard level. Thus, meeting $\wedge T F$ performance requirement should not be a problem. The most severe subsystem requirement is to control the jitter of the image at the Ronchi ruling to 0.01 arcsec in a bandwidth between one-half, and two times the ruling frequency. Theoretical analyses of vibration isolation 
performance based on the above units assuming a very conservative vibration level of $10^{-2} \mathrm{~g}$ input from the Space $S$ tation and using the most sensitive modes from the NASTRAN model for the ATF structure show that vibration isolation performance on the order of $50 \mathrm{~dB}$ is required. This appears to be well within the capabilities of systems tested on the ground.

\section{Power and harness}

Primary electrical power supplied by the Space Station to ATF will be through the power electronics unit (power interface unit) which provides voltage regulation, power switching, and under/over-voltage control. The power subsystem will have the capability to switch individual ATF units on or off. This power control unit will be designed specifically for ATF but will incorporate standard voltage regulation boards designed and qualified for the Space Station program. All the harnesses for ATF will use standard flight design with connectors designed and qualified for on-orbit mating and demating as required.

\section{Operations}

The study examined the operations requirements for ATF and defined a strawman approach based on experience gained from the Pioneer operations at Ames Research Center and the Solar Mesospheric Explorer (SME) operations at the University of Colorado. Basic assumptions for the study were:

1. ATF will receive high priority for use of Space Station resource during assembly and initial checkout.

2. During normal operations, ATF will need a minimum of planning coordination with the Space Station and with other payloads.

3. ATF operation interruptions caused by Space Station and other sources will be infrequent or short.

4. Space Station will provide warning and all-clear signals for disturbance events.

Based on these assumptions, the basic functional approach to operations and an outline of the initial mission events (assembly, checkout and initial operation), normal operations, and approach to anomalous events or conditions has been defined. Operations defined by this study appear straightforward. Normally ATF data will be transmitted to the ground and the ATF operations center on a near real-time basis, therefore the receipt and logging of the data will be a continuous full-time task. The baseline operations plan requires generation and updating of the ATF onboard command sequence once per week. Observation and facility management sequence generation can be accomplished on a regular 5-day per week, single-shift basis.

A straightforward approach is also planned for anomalous or failure conditions. This is possible because the scientific data collection is not time-critical and there are no mission events which are time-critical except when pointing into the sun or a loss of power (which could lead to critical mispointing). These critical conditions are handled automatically by closing the facility cover and waiting for ground intervention for recovery. In the event of short term (hours) noncritical interruptions, the plan is to continue the existing sequence. The portion of the data lost during the down-time would be rescheduled in a subsequent sequence.

\section{Observing analysis}

The study analyzed the capability of the proposed mission and hardware to provide the viewing and tracking time for the required planetary detection observations. A tracking and observation strategy computer simulation program ${ }^{9}$ was developed for this analysis. A baseline set of 127 possible t.aget stars, including a several different star types, was used in the analysis. Viewing constraints were described earlier. Figure 9 shows a typical viewing window when all of the constraints are considered. The results of the analysis for a 1 -yr period of operation show that nearly $80 \%$ of the time is spent actually looking at a target, and that the observation time can be relatively evenly distributed over the stars. A parametric examination showed the total amount of viewing time to be insensitive to changes in constraints, although significant variations in overall efficiency can result from changes to the set of target stars.

\section{Conclusions}

The ATF Preliminary System Definition Study has defined a relatively straightforward strawman system which can meet the requirements for a extrasolar planetary systems search and study. The facility will be useful for a number of other astrophysics investigations, including measurements of stellar distances and motions. The basic measurement approach proposed for ATF has been proven by ground based investigations conducted at the University of Pittsburgh's Allegheny Observatory.

The facility is designed as a Space Station payload, using the standard Space Station services plus one of the Space Station CPS. A major portion of the engineering system hardware in the strawman design is either already flight qualified or will be designed and qualified for the Space Station program. The strawman MAP of the FPI design is based on an instrument presently in use at University of Arizona's Steward Observatory.

A basic operations approach was identified. This included the approach to launch, initial on-orbit assembly and checkout, normal operations, and the response to anomalous conditions or failures. The results of the study showed that ATF operations should be comparable in scope to Pioneer operations at NASA Ames, or SME at the University of Colorado.

Mission analysis shows the basic viewing required for planetary detection can be accomplished in approximately two-thirds of the total viewing time. This leaves on the order of $2000 \mathrm{hr} / \mathrm{yr}$ for other astrophysics investigations.

The study did not identify any feasibility issues or any areas where basic technology development is required. 
The study concludes that the concept is ready for a detailed indepth engineering design study. As the Space Station configuration is finalized, accommodations and compatibility for early science operation of the ATF can be secured with the early definition of the ATF.

\section{References}

1. Oliver, B. M., ed., Project Cyclops, A Design Study of a System for Detecting Extraterrestrial Intelligent Life, NASA CR 114445 , 1971.

2. Greenstein, J., Chairman, "Minutes--First Workshop on Extrasolar Planetary Detection," Hale Observatory, 1976.

3. Greenstein, J, Chairman,"Minutes--Second Workshop on Extrasolar Planetary Detection," Hale Observatory, 1976.

4. An Assessment of Ground-Based Techniques for Detecting Other Planetary Systems--Vol. I: Overview and Vol, II: Position Papers, NASA CP-2124, 1980.

5. Black, D. C., Chairman, "Toward the Detection and Study of Other Planetary Systems--Science Workshop Report to NASA," 1983.

6. Black, D. C., ed., Project Orion. Design Study of a System for Detecting Extrasolar Planets, NASA SP-436, 1980.

7. "Comparative Feasibility Study of Two Concepts for a Space-Based Astrometric Satellite," LMSC-D870885, 1982.

8. "Planetary Detection Astrometric Telescope Study," LMSC-D866237, 1984.

9. Mascy, A. C., Nishioka, K., Jorgensen, H., and Swenson, B., "Detection of Planetary Systems From Space Station-A Star Observation Strategy," AIAA Paper No. 87-0317, 1987.

Table 1. Potential Systematic Effects

\begin{tabular}{|c|c|}
\hline Effects & Solution \\
\hline Optical Surface Variations & $\begin{array}{l}\text { Use One Optical Surface in Entrance } \\
\text { Pupil }\end{array}$ \\
\hline Coma & Optimized Ruling - 2 Color Bands \\
\hline Changes of Mirror Coating & $\begin{array}{l}\text { Overcoat to Hold Within Specs and } \\
\text { Protect from Contamination }\end{array}$ \\
\hline Non-uniform Ruling Deformation & $\begin{array}{l}\text { Material Selection and Statistical } \\
\text { Testing }\end{array}$ \\
\hline $\begin{array}{l}\text { Transmission Changes of Secondary Optics } \\
\text { (Rullng, Optical Fibers, etc.) }\end{array}$ & Materlal Selection \\
\hline Fleld Crowding & $\begin{array}{l}\text { Examination of Fields for Optical } \\
\text { Doubles }\end{array}$ \\
\hline Unknown Nonlinear Reference Star Motions & $\begin{array}{l}\text { Selection of Distant Reference Stars } \\
\text { and Continuous Modeling }\end{array}$ \\
\hline
\end{tabular}


Table 2. Random Errors



- Integration Time Needed to Reach a Given Accuracy is Increased by Random Errors.

*F = Contribution to the Integration Time Enhancement Factor; Total Time Enhancement is the Sum of the Individual F's.

* To Be Determined.

Notes: (a) The Design Requirement Corresponding to $E \ll 1$ is Feasible.

(b) Included in Jitter.

(c) With Proper Selection of Fields and Reference Stars This will be a Negligible Error (F $<1)$.

Table 3. Light-Loss Effects

\begin{tabular}{lll}
\hline Source of Information Loss & Type of Loss & Throughput \\
\hline A. Grating Rejection & Light & 0.25 \\
B. Mask for Grating-Shadow & Light & 0.75 \\
C. Grating Intrinsic & Information & 0.50 \\
D. Loss in Optics & Light & 0.50 \\
E. Detector Quantum Inefficiency & Light & 0.10 \\
F. One-Dimensional Engine & Information & 0.50 \\
G. Operational Interruptions & Information & 0.50 \\
& Total Throughput & 0.00117 \\
\hline
\end{tabular}

- Integration Time Needed to Reach a Given Accuracy is Increased by Light and Other Information Losses.

- Integration Time Increased by 1/Throughput $=853$ 
Table 4. Observation Time Calculations

\begin{tabular}{|c|c|c|c|}
\hline $\begin{array}{l}\text { Galactic } \\
\text { Latitude }\end{array}$ & $\begin{array}{l}\text { Ideal Integration Time } \\
\text { (Minutes) }\end{array}$ & $\begin{array}{c}\text { Average Observat } \\
\text { (Hours) }\end{array}$ & ion Time \\
\hline 0 & 0.030 & 0.58 & \\
\hline 10 & 0.57 & 10.98 & \\
\hline 20 & 1.37 & 26.6 & Most Observations \\
\hline 30 & 2.19 & 42.4 & at Low Lati tudes \\
\hline 40 & 3.12 & 60.4 & \\
\hline 50 & 4.12 & 80.6 & \\
\hline 60 & 5.00 & 96.6 & \\
\hline 70 & .5 .95 & 115 & \\
\hline 80 & 6.25 & 120.8 & \\
\hline 90 & 6.58 & 127.2 & \\
\hline
\end{tabular}

Total Observation $=$

Integration Time for Ideal System

$x$

Time Factor for Light and Information Losses (853)

$X$

Time Factor for Random Errors (1.36)

For ATF: Overall Time Factor $=853 \times 1.36=1160$

Observation Time $=1160 \times$ Ideal Integration Time

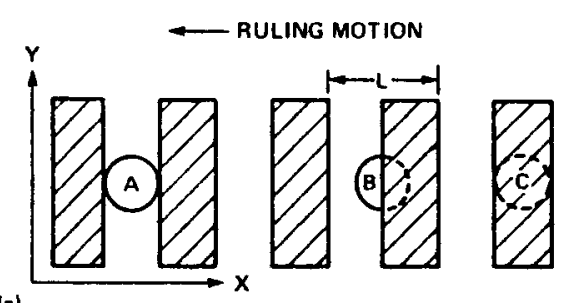

(a)

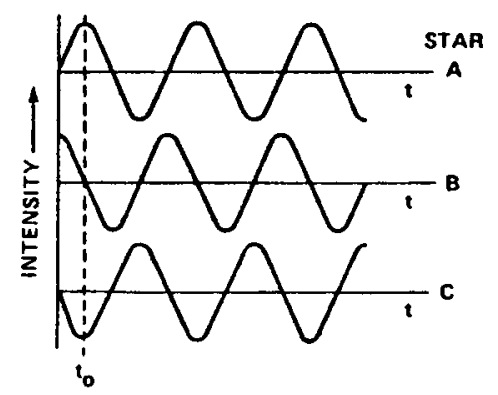

Figure 1. Measurement approach. 


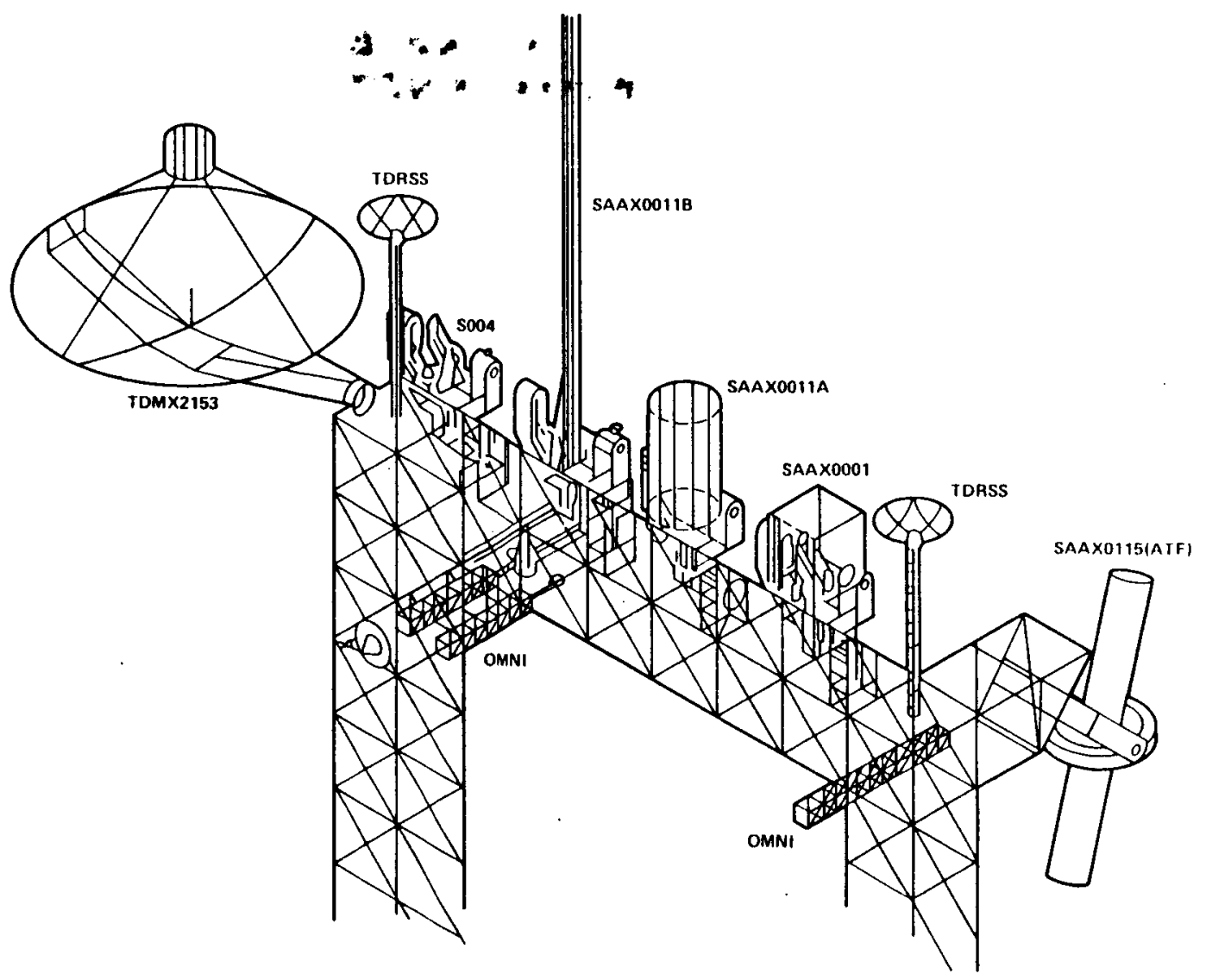

Figure 2. ATF mounted on Space Station (baseline design).

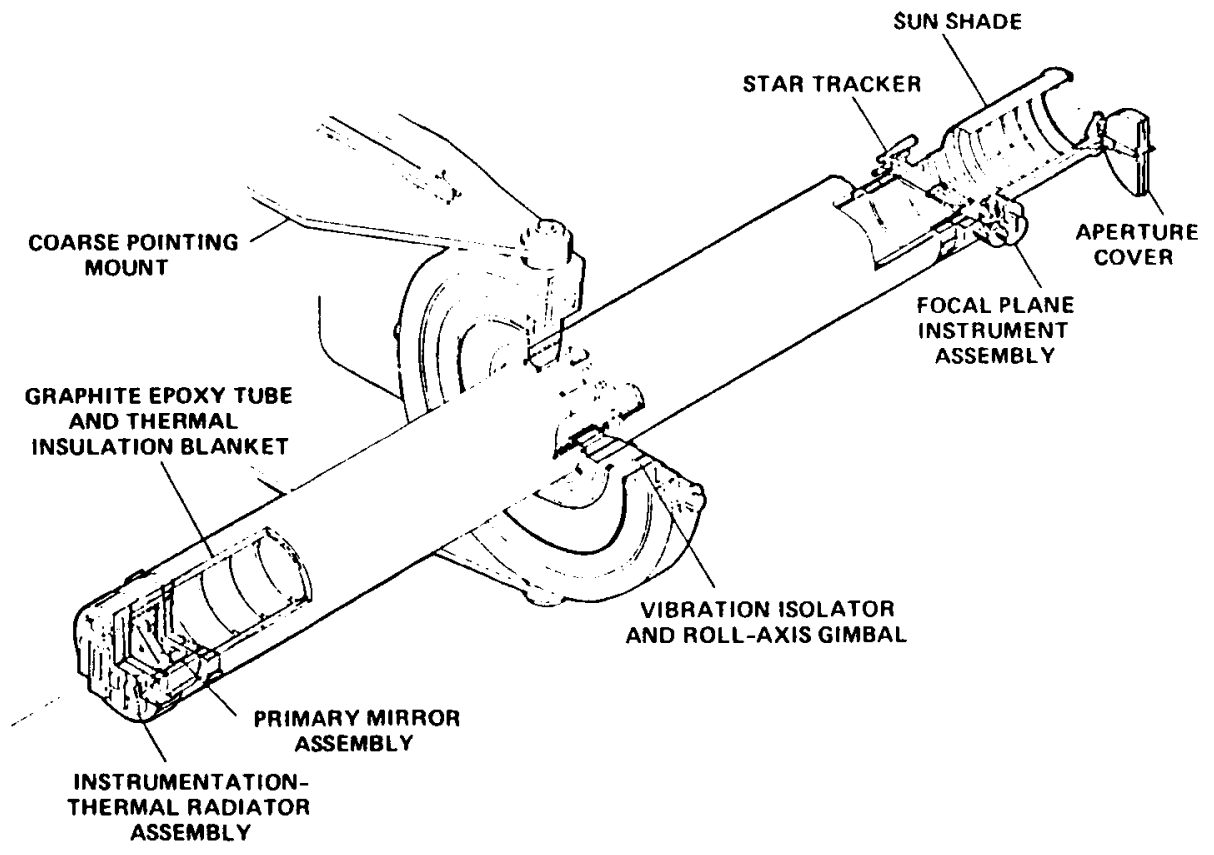

Figure 3. Astrometric telescope facility (ATF). 


\section{ORIGINAL PAGE ES
Or MOOR QUALTY}

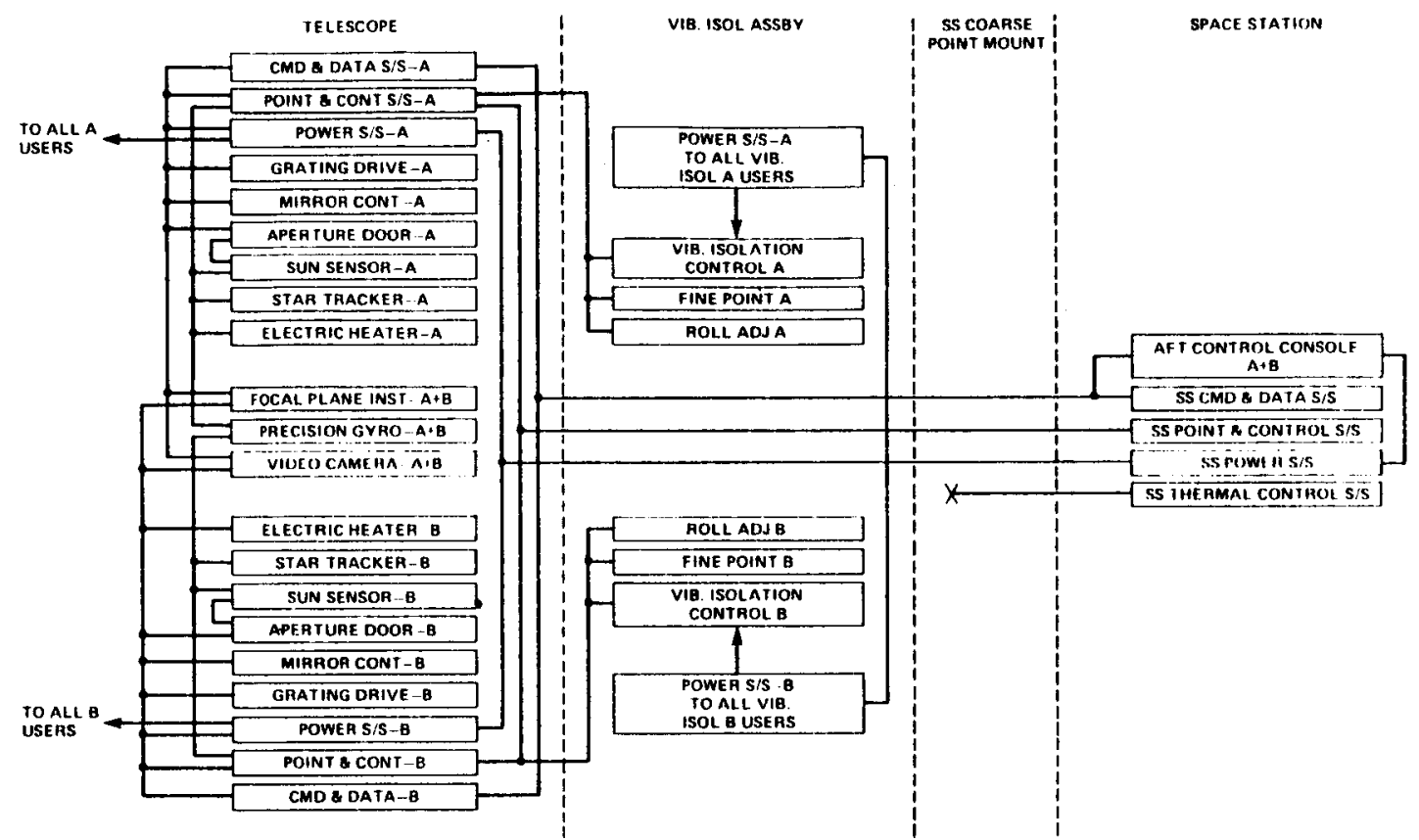

Figure 4. ATF block diagram. 

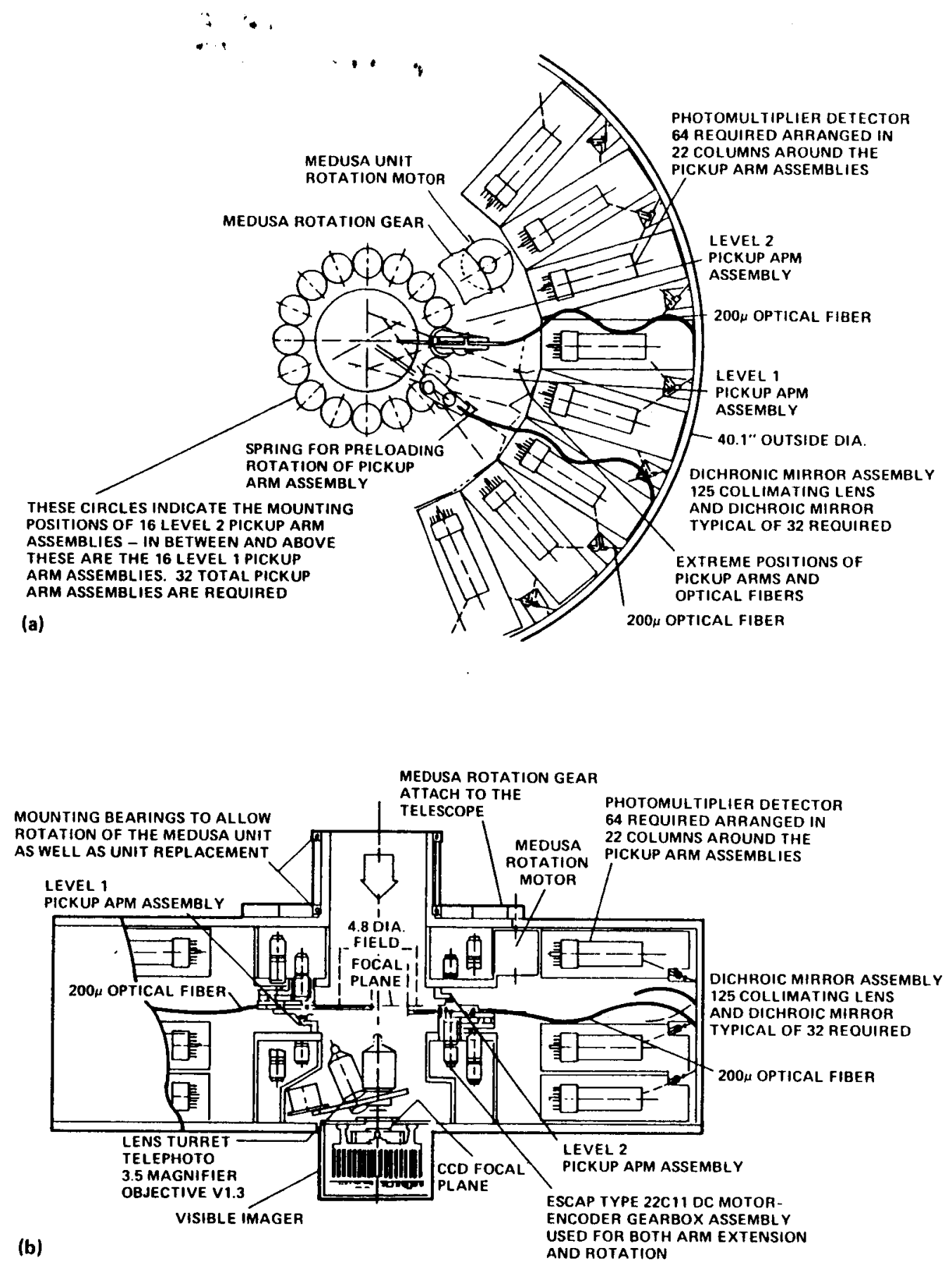

Figure 5. ATF focal plane instrument.
a. "Medusa" side view.
b. "Medusa" top view. 




Figure 6. Ronchi ruling assembly.

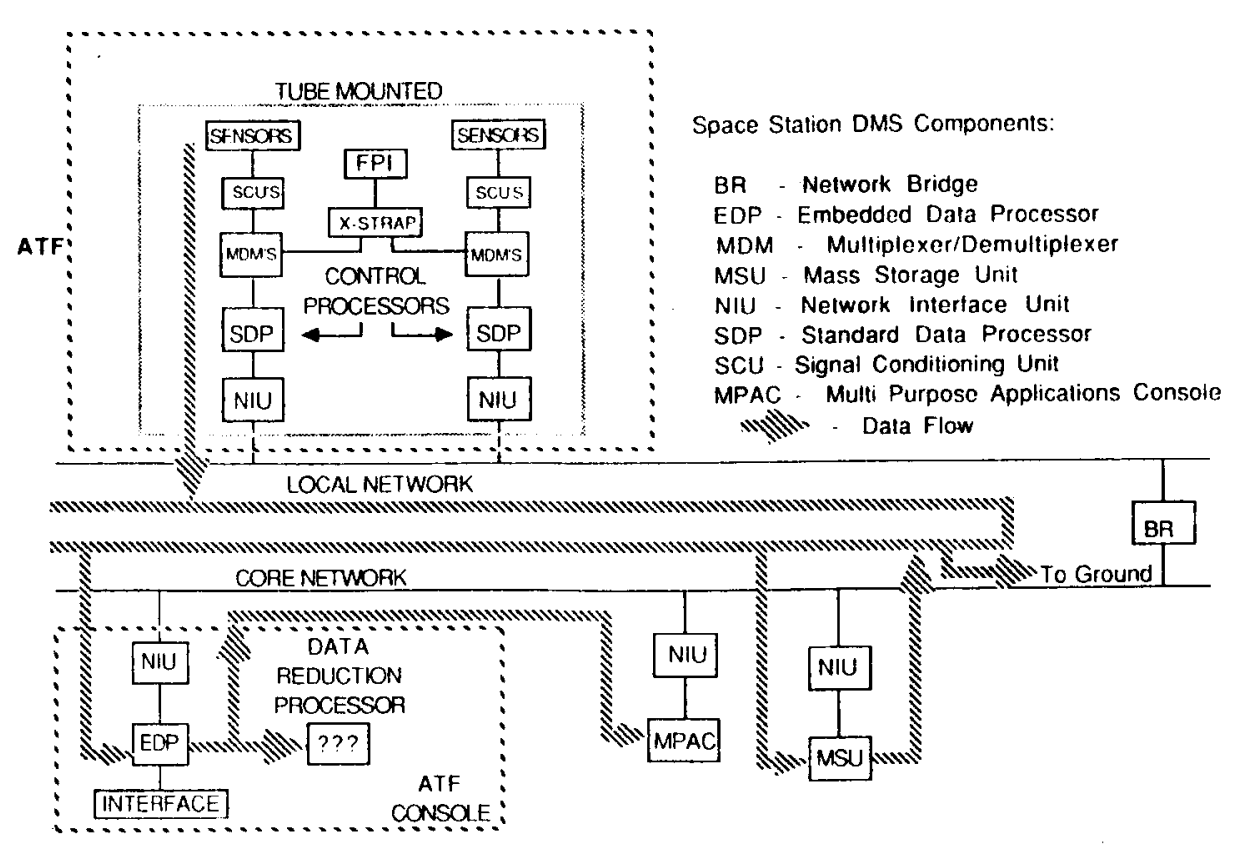

Figure 7. Command and data subsystem. 


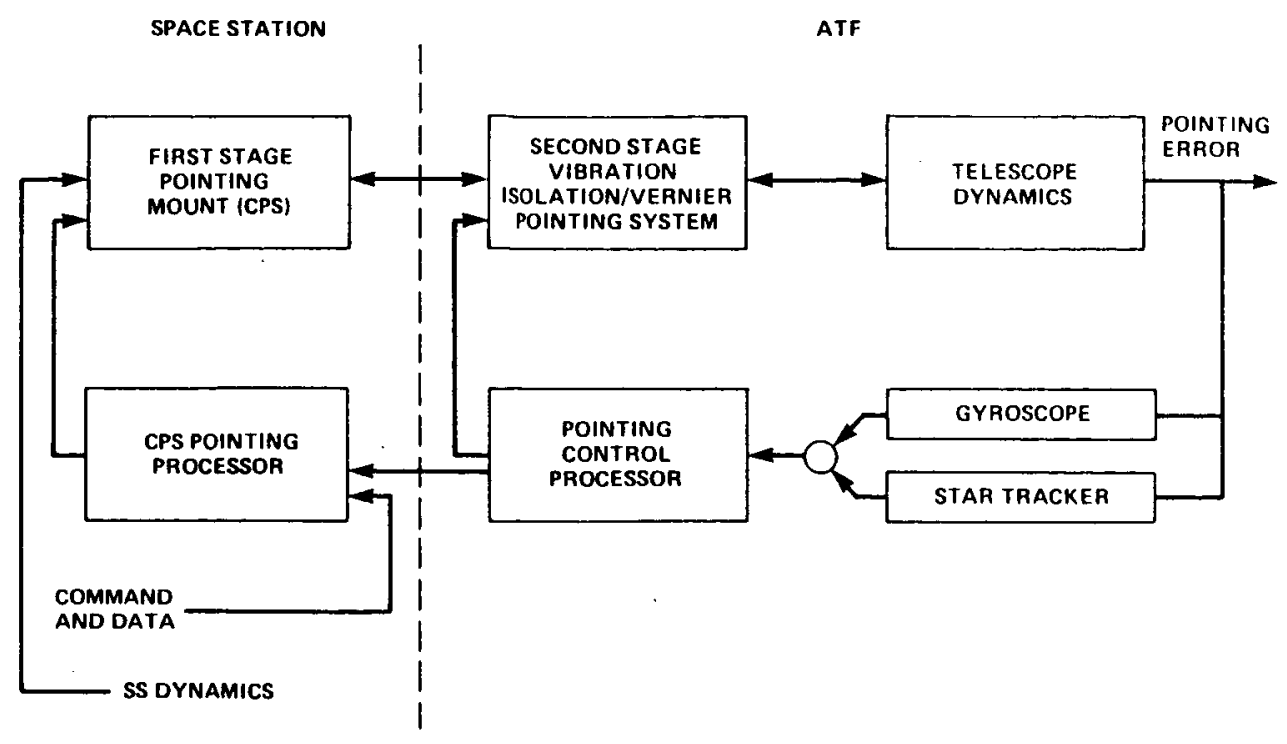

Figure 8. Pointing and control subsystem.

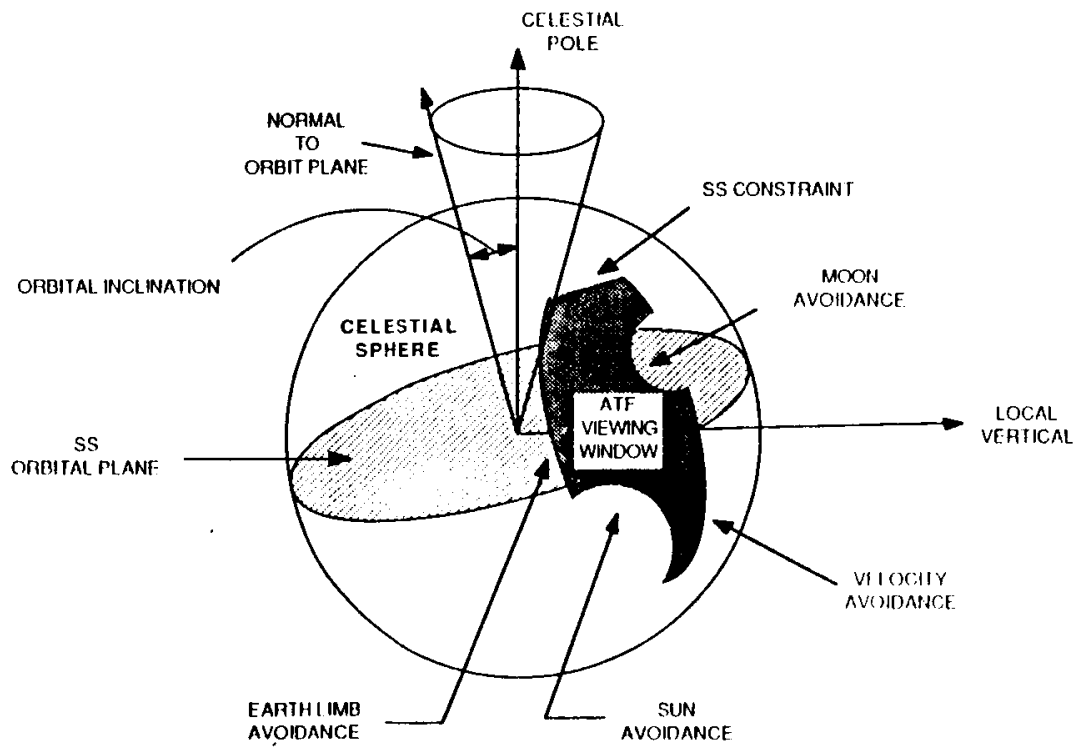

Figure 9. ATF viewing window. 


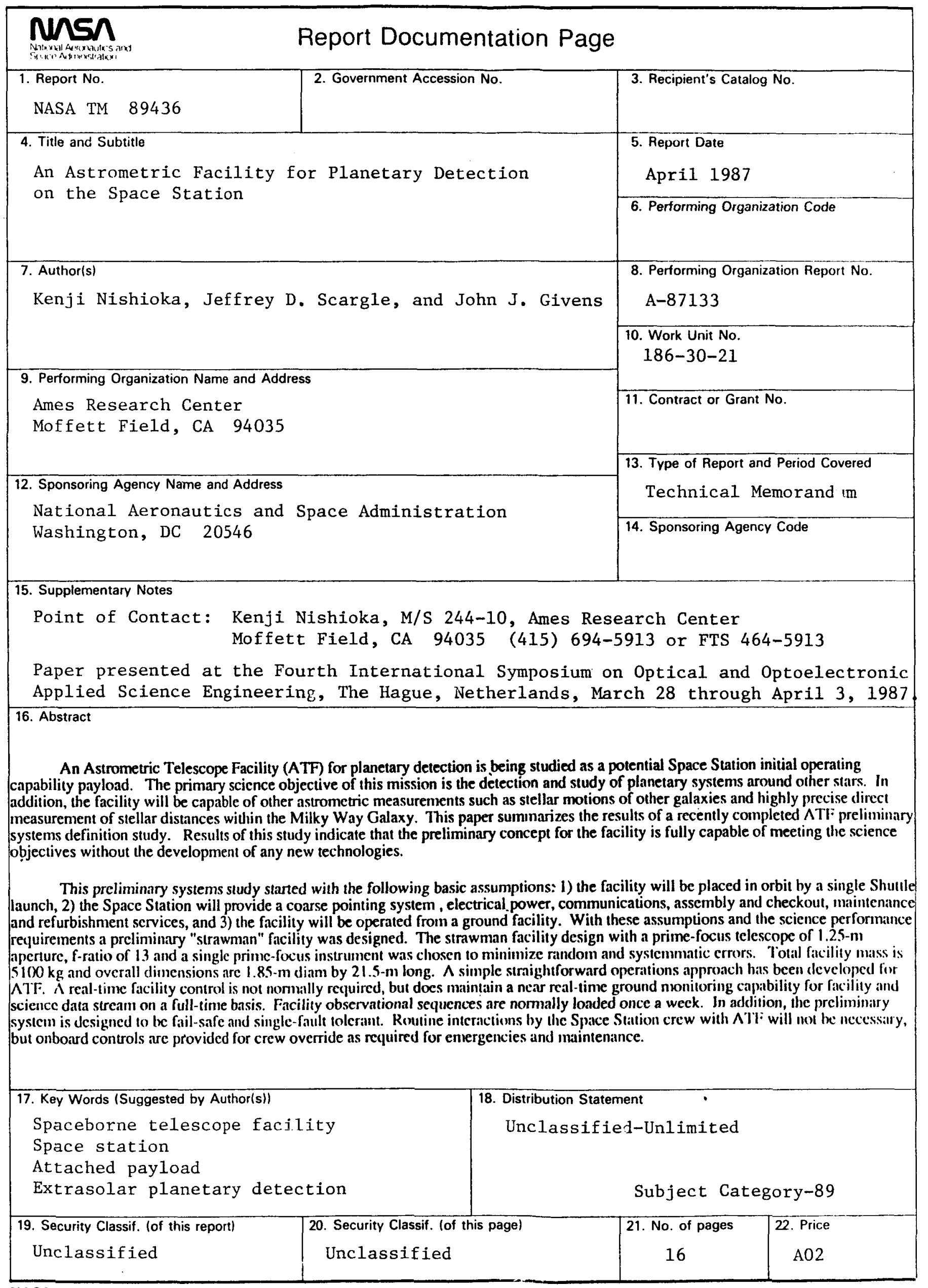

